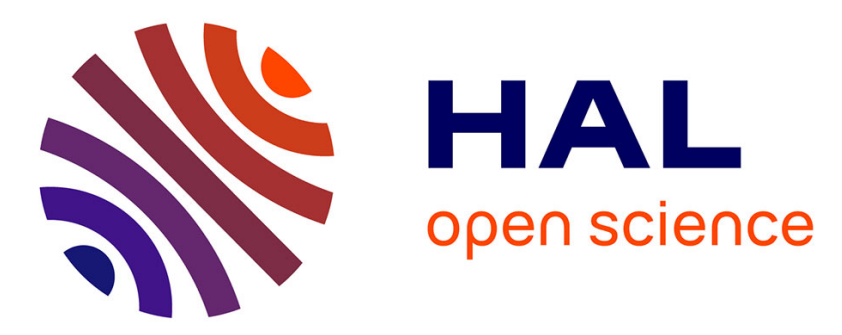

\title{
Energie critique des grains Nd2Fe14B monodomaines dans les aimants amincis
}

J. Bras, K. Biyadi, M. Fagot, J. Degauque

\section{To cite this version:}

J. Bras, K. Biyadi, M. Fagot, J. Degauque. Energie critique des grains Nd2Fe14B monodomaines dans les aimants amincis. Journal de Physique IV Proceedings, 1992, 02 (C3), pp.C3-113-C3-117. 10.1051/jp4:1992317 . jpa-00251522

\section{HAL Id: jpa-00251522 https://hal.science/jpa-00251522}

Submitted on 1 Jan 1992

HAL is a multi-disciplinary open access archive for the deposit and dissemination of scientific research documents, whether they are published or not. The documents may come from teaching and research institutions in France or abroad, or from public or private research centers.
L'archive ouverte pluridisciplinaire HAL, est destinée au dépôt et à la diffusion de documents scientifiques de niveau recherche, publiés ou non, émanant des établissements d'enseignement et de recherche français ou étrangers, des laboratoires publics ou privés. 


\title{
Energie critique des grains $\mathrm{Nd}_{2} \mathrm{Fe}_{14} B$ monodomaines dans les aimants amincis
}

\author{
J. BRAS, K. BIYADI, M. FAGOT et J. DEGAUQUE
}

Physique des Solides (URA 74), Départ Physique INSAT, Av. de Rangueil, 31077 Toulouse cedex, France

\begin{abstract}
The magnetic domain structure of $\mathrm{Nd}-\mathrm{Fe}-\mathrm{B}$ based crystalline grains is studied by transmission electron microscopy. The action of the objective lens magnetic field on the sample induces magnetization reversals. The experimental conditions of nucleation and saturation are compared with theoretical curves of magnetic energy of single domain particles.
\end{abstract}

\section{1 - Introduction.}

L'observation d'échantillons magnétiques dans les microscopes électroniques en transmission courants provoque une action du champ magnétique de l'objectif sur l'objet et généralement une modification de sa structure magnétique. Dans le cas des matériaux à très forte anisotropie magnétocristalline uniaxiale cette action peut être approchée théoriquement en fonction des paramètres expérimentaux, et notamment l'énergie libre d'un grain monodomaine isolé d'orientation connue peut être calculée numériquement. Ces résultats théoriques sont comparés ici avec les résultats expérimentaux de l'étude par microscopie de Lorentz de la structure en domaines magnétiques des aimants NéodymeFer-Bore.

\section{2 - Conditions expérimentales}

Deux séries d'alliages ont été étudiées. La première, de composition atomique voisine de $\mathrm{Nd}_{13} \mathrm{Fe}_{81} \mathrm{~B}_{5} \mathrm{Al}$ a été élaborée au Centre d'Etudes Nucléaires de Grenoble par A. CHAMBEROD \& Coll. par trempe sur roue avec des vitesses de 20 et $22,5 \mathrm{~m} / \mathrm{s}$. Les études ont porté à la fois sur des rubans bruts de trempe (type I), sur des aimants compactés à chaud (type II) et sur des aimants orientés par déformation à chaud (type III). La deuxième série a été élaborée par la société UGIMAG à partir de poudres orientées compactées et frittées. Elle comporte deux familles de composition atomique $\mathrm{Nd}_{13,6} \mathrm{Dy}_{1,4} \mathrm{Fe}_{76,6} \mathrm{Nb}_{0,7} \mathrm{Al}_{1} \mathrm{~B}_{6,7}$ et $\mathrm{Nd}_{13,5} \mathrm{Dy}_{1,5} \mathrm{Fe}_{67} \mathrm{Co}_{5} \mathrm{~V}_{4} \mathrm{~B}_{8} \mathrm{Al}_{1}$. Les échantillons sont désaimantés thermiquement, amincis mécaniquement puis par bombardement ionique et conservés sous vide pour diminuer l'oxydation.

L'orientation cristalline de chaque grain étudié est déterminée au préalable, notamment l'angle $\alpha$ entre l'axe $\mathrm{C}$ d'anisotropie cristalline et la normale $\mathrm{N}$ à la lame mince. Grâce au porte-objet inclinaison-rotation, on fait alors tourner l'échantillon dans son plan pour amener l'axe C perpendiculaire à l'axe d'inclinaison : les conditions optimales [1] de contrôle de l'action du champ $\mathbf{H}$ de la lentille objectif sur l'objet sont ainsi réalisées. L'angle $\theta$ entre $H$ et $C$ pour un angle d'inclinaison $T$ est alors $\theta=\alpha+T$

Les domaines magnétiques sont visualisés par microscopie de Lorentz à partir des déviations opposées du faisceau électronique crées par leur aimantation. Le contraste est 
obtenu soit en occultant une partie du faisceau. (méthode de Foucault), soit par défocalisation (méthode de Fresnel).

\section{3 - Energie d'un grain monodomaine dans un champ extérieur}

Considérons un grain cristallin monodomaine aminci, assimilé à un sphèroïde aplati d'axe de révolution a (voir figure 1 ).

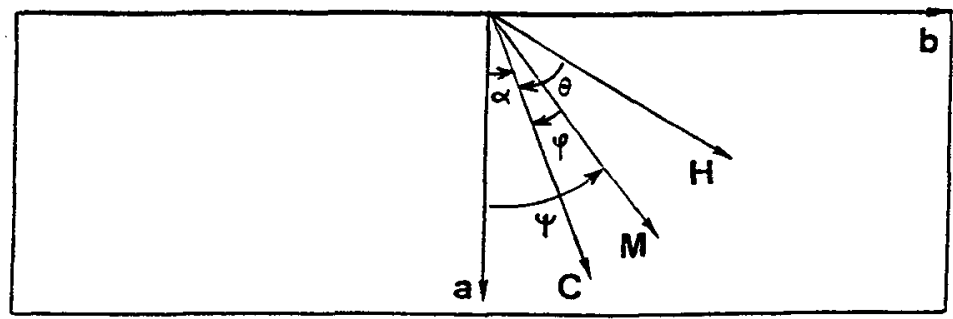

Figure 1 : paramètres de l'énergie magnétique d'une particule monodomaine

Son énergie libre $\mathbf{F}_{\mathbf{H}}$ est la somme de l'énergie d'anisotropie cristalline, de l'énergie magnétique et de l'énergie du champ démagnétisant. Elle est donnée par [2,3] :

$$
\mathrm{F}_{\mathrm{H}}=1 / 2 \mu_{o} \mathrm{M}_{\mathrm{s}}^{2}\left(\mathrm{~N}_{\mathrm{a}} \cos ^{2} \psi+\mathrm{N}_{\mathrm{b}} \sin ^{2} \psi\right)+\mathrm{K} \sin ^{2} \varphi-\mu_{o} \mathrm{HM}_{\mathrm{s}} \cos (\theta-\varphi)
$$

avec : $\psi=(\mathbf{a}, \mathbf{M}) \quad, \quad \varphi=(\mathrm{M}, \mathrm{C}) \quad, \quad \theta=(\mathbf{H}, \mathrm{C})$

Posons : $\mathrm{A}=\mathrm{M}_{\mathrm{s}} / 2 \mathrm{H} \quad, \quad \mathrm{B}=\mathrm{K} / \mu_{\mathrm{o}} \mathrm{M}_{\mathrm{s}} \mathrm{H} \quad, \quad \alpha=(\mathrm{a}, \mathrm{C})$

et $\quad \mathrm{Z}=\mathrm{N}_{\mathrm{b}}=\mathrm{N}_{\mathrm{c}} \quad$ avec $\mathrm{N}_{\mathrm{a}}+\mathrm{N}_{\mathrm{b}}+\mathrm{N}_{\mathrm{c}}=\mathbf{1}$

et appelons E l'énergie réduite :

$$
\mathrm{E}=\mathrm{F}_{\mathrm{H}} / \mu_{o} \mathrm{HM}_{\mathrm{S}}=\mathrm{A}\left[\mathrm{Z}+(1-3 \mathrm{Z}) \cos ^{2}(\alpha-\varphi)\right]+\mathrm{B} \sin ^{2} \varphi-\cos (\theta-\varphi)
$$

Dans le cas du microscope électronique JEOL $200 \mathrm{CX}(\mathrm{H}=0,6 \mathrm{MA} / \mathrm{m})$, avec les valeurs $\mathrm{K}$ $=4,5 \mathrm{MJ} / \mathrm{m}^{3}$ et $\mathrm{M}_{s}=1,27 \mathrm{MA} / \mathrm{m}$ de la phase $\mathrm{Nd}_{2} \mathrm{Fe}_{14} \mathrm{~B}$, et compte tenu de l'épaisseur très faible des grains, le premier terme de $\mathrm{E}$ (anisotropie de forme) ne peut pas être négligé. Le calcul de l'angle $\varphi$ qui minimise l'énergie doit donc être effectué numériquement.

Les paramètres $A$ et $B$ sont caractéristiques du matériau et du champ et restent donc fixes.(avec les valeurs ci-dessus, on $a A=1,1$ et $B=4,7$ ). Les paramètres $\alpha$ et $Z$ sont caractéristiques de chaque grain étudié. Pour un grain donné il reste donc une seule variable expérimentale $\theta$ liée à l'inclinaison de l'échantillon dans le microscope.

Pour chaque valeur de $\theta$ un programme de calcul recherche automatiquement les valeurs de $\varphi$ qui minimisent $E$. Nous désignons par $\varphi_{1}$ et $\varphi_{2}$ les deux valeurs respectivement voisines de zéro et de $\pi$ qui correspondent à l'aimantation directe (aimantation dans le 
sens de C) et à l'aimantation inverse. Par convention, est choisie comme positive la direction de $\mathrm{C}$ qui va dans le même sens que les électrons lorsque $\mathrm{T}=0$.

Le premier résultat de ce calcul concerne la direction de l'aimantation : on pourra toujours la confondre avec celle de l'axe $C$ car dans les cas théoriques les plus défavorables elle s'en écarte au maximum de 12 degrés et en pratique de quelques degrés seulement.

\section{4 - Energie limite des grains monodomaines. Confrontation avec l'expérience.}

Les figures 2 et 3 montrent les courbes théoriques de l'énergie réduite $\mathrm{E} \varphi_{1}$ de onze grains (a à k) dans lesquels les conditions d'apparition de domaines d'aimantation inverse ont été étudiées en détail en vue d'une comparaison entre les résultats expérimentaux et les prévisions théoriques du modèle du grain isolé.

Chaque courbe correspond à un grain dont les caractéristiques, $\alpha$ (en degrés) et Z, sont indiquées à droite du symbole. $\alpha=(\mathrm{N}, \mathrm{C})$ est déterminé expérimentalement grâce aux diagrammes de microdiffaction et $\mathrm{Z}$ est relié au rapport $\mathrm{b} / \mathrm{a}$ entre les dimensions du grain (son diamètre moyen $b$, directement mesurable, et son épaisseur moyenne a, estimée entre 50 et100nm à partir des contrastes observés) : les valeurs de Bozorth [4] ont été utilisées (typiquement $Z=0,05-0,1-0,25-0,33$ pour $b / a=15-6,7-2,5-1$ ).

Les courbes théoriques, donnent les valeurs de $E$ pour des valeurs de $\theta$ variant de $-100^{\circ}$ $\grave{a}+100^{\circ}$. Elles montrent que l'influence de $\alpha$ pour une orientation du champ donnée, par exemple pour $\theta=0$, est presque aussi importante $(\Delta E=0,7)$ que celle d'une rotation $d u$ champ de $90^{\circ}$ pour $\alpha$ égal à $28^{\circ}(\Delta \mathrm{E}=0,9)$.

L'influence de $Z$ est visible en comparant par exemple les courbes des grains $d\left(\alpha=62^{\circ}, Z\right.$ $=0,19)$ et $\mathrm{j}\left(\alpha=-66^{\circ}, \mathrm{Z}=0,0055\right):$ minima égaux respectivement à $-0,7$ et $-0,85$.

Le domaine de variation de $\theta$ choisi pour les courbes $\mathrm{E}_{\varphi 1}$ est en général suffisant pour rendre compte des situations expérimentales rencontrées : d'une part, l'angle d'inclinaison dépasse rarement $45^{\circ}$, ce qui limite le domaine de variation de $\theta$ à $\Delta \theta=90^{\circ}$, et d'autre part, les règles de symétrie suivantes sont appliquées :

$$
\begin{gathered}
\mathrm{E}(\alpha, \theta)=\mathrm{E}(-\alpha,-\theta) \\
\varphi(\alpha, \theta)=-\varphi(-\alpha,-\theta) \\
\mathrm{E}_{\varphi_{2}}(\alpha, \theta)=\mathrm{E}_{\varphi_{1}}(\alpha, \theta-\pi) \operatorname{avec} \varphi_{2}=\pi+\varphi_{1}
\end{gathered}
$$

Lors du renversement d'aimantation d'un grain à partir d'une situation monodomaine, on suit sur la courbe (partie inférieure) l'augmentation d'énergie en fonction de $\theta$ jusqu'à une valeur limite (repère $G$ ) à partir de laquelle le modèle n'est plus valable puisque le système gagne de l'énergie en se divisant en domaines Cette limite est par exemple $\mathrm{E}_{\boldsymbol{\varphi}}$, atteinte pour un angle $\theta_{11}$. Si la variation de $\theta$ se poursuit dans le même sens, l'aimantation moyenne du grain s'annule et la saturation en aimantation inverse se produit pour $\theta_{12}$. L'exemple suivant illustre le suivi d'une situation expérimentale sur la courbe théorique correspondante.

\section{Exemple du grain $k$ (figure 3)}

$$
\alpha=(\mathrm{N}, \mathrm{C})=-77^{\circ} \quad \mathrm{b} / \mathrm{a} \sim 2000 \mathrm{~nm} / 100 \mathrm{~nm}=20 \text {, ce qui donne } \mathrm{z}=0,03
$$

$\mathrm{T}$ varie de $+45^{\circ} \mathrm{à}-45^{\circ}$ donc $\theta$ varie de $-32^{\circ} \mathrm{a}-122^{\circ}$.

Sur la partie gauche de la courbe, l'expérience débute par une situation monodomaine à $\theta$ $=-32^{\circ}$ (flèche); jusqu'à $\theta=-82^{\circ} l^{\prime}$ 'énergie $E_{\varphi 1}$ croît de $-0,76$ à la valeur limite $-0,07$.(repère

G1 : énergie de germination). Au-dessus de $\theta=-82^{\circ}$, des domaines inverses sont présents, le modèle n'est plus valable. Pour $\theta=-92^{\circ}$, le grain est à nouveau saturé avec 


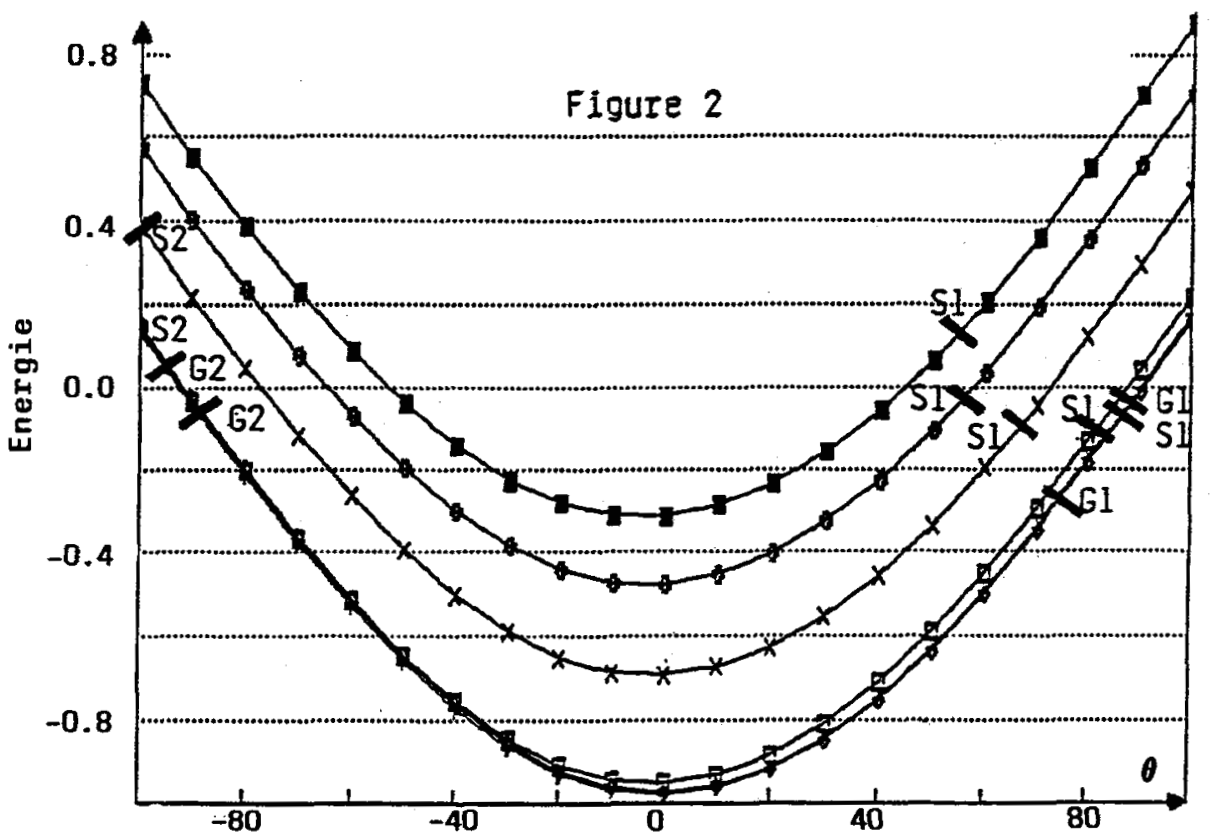

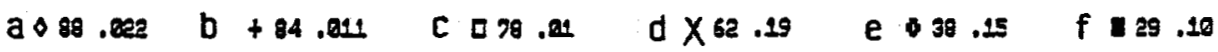

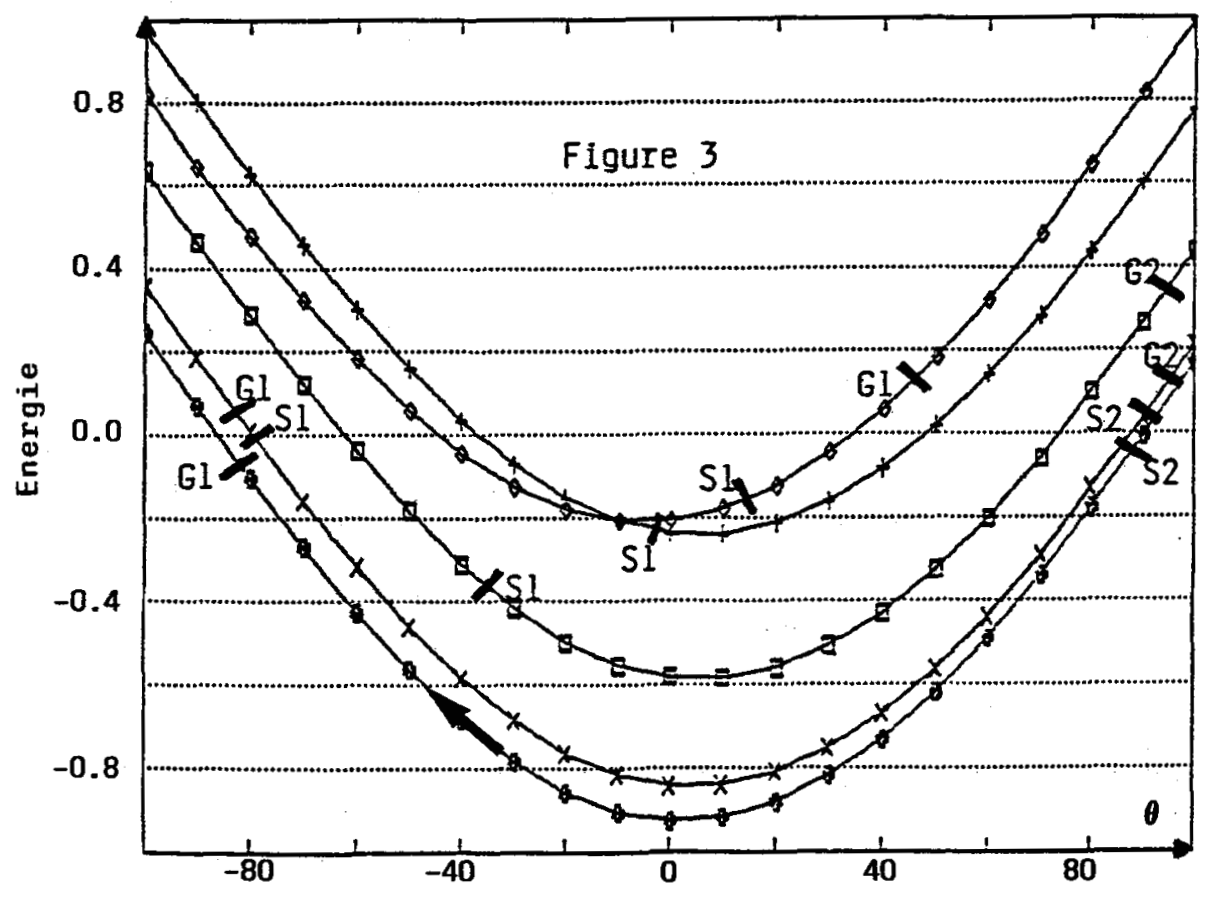

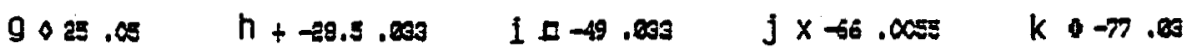

Figures 2 et 3 (voir texte $\$ 4$ ) : au-dessus des limites $S$ et $G$, le modèle n'est plus valable. 
$\varphi_{\mathbf{2}} \sim \pi$, donc avec une énergie $\mathrm{E} \varphi_{2}$. La troisième règle de symétrie nous permet de lire la valeur de $\mathrm{E}_{\varphi_{2}}$ sur la partie droite de la courbe $\mathrm{E}_{1}$ :

$$
E \varphi_{2}(-77 ;-92)=E \varphi_{1}(-77,+88)
$$

soit une valeur de l'énergie de saturation $\mathrm{E}_{\varphi_{2}}=-0,04$ (repère S2)

Les limites expérimentales sont indiquées sur la courbe de chaque grain par un trait perpendiculaire à la courbe. Les lettres G (germination) ou $S$ (saturation) et les chiffres 1 $\left(\varphi_{1}\right)$ ou $2\left(\varphi_{2}\right)$ précisent la nature de la transition. Les valeurs des énergies ainsi déterminées sont présentées dans le tableau 1

\begin{tabular}{|c|c|c|c|c|c|c|c|c|c|c|c|}
\hline grains & a & b & c & d & e & f & g & h & i & i & k \\
\hline & $\mathbf{8 8}$ & $\mathbf{8 4}$ & $\mathbf{7 8}$ & $\mathbf{6 2}$ & $\mathbf{3 8}$ & $\mathbf{2 8}$ & $\mathbf{2 5}$ & $-28,5$ & -49 & -66 & $-\mathbf{7 7}$ \\
\hline G1 & & & & & & & $\mathbf{0 , 1 2}$ & & & $\mathbf{0 , 0 4}$ & $-\mathbf{0 , 0 7}$ \\
\hline S1 & $-0,06$ & & $-0,1$ & $-0,09$ & $-0,03$ & $\mathbf{0 , 1 2}$ & $-0,16$ & $-0,25$ & $-\mathbf{0 , 3 7}$ & $\mathbf{0 , 0 4}$ & \\
\hline G2 & $-0,05$ & & $\mathbf{0 , 0 4}$ & & & & & & $\mathbf{0 , 3 2}$ & $\mathbf{0 , 1 3}$ & \\
\hline S2 & & $\mathbf{0 , 0 4}$ & & $\mathbf{0 , 4 2}$ & & & & & & $\mathbf{0 , 0 6}$ & $\mathbf{- 0 , 0 4}$ \\
\hline
\end{tabular}

Tableau $1:$ Energies relevées sur les courbes des figures 2 et 3.

G1 (G2) : germination au sein d'un grain d'aimantation directe (inverse)

S1 (S2) : saturation du grain avec une aimantation directe (inverse)

\section{5 - Discussion}

Le résultat le plus évident est la validité remarquable du modèle du grain monodomaine isolé appliqué à une situation expérimentale complexe : les valeurs des énergies limites se situent nettement autour d'une valeur moyenne voisine de zéro. Les écarts à cette valeur moyenne s'interprètent par l'influence des grains voisins, plus forte pour les grains de petite taille (exemple du grain d), mais aussi dans certains grains plus grands (grains i et $\mathrm{h}$ en interaction mutuelle), ce qui confirme des résultats antérieurs $[5,6]$. L'influence primordiale de $\alpha$ sur les conditions d'apparition des domaines prévue par le modèle est confirmée par l'expérience : notamment les grains dans lesquels $\alpha$ est inférieur à 25 degrés ne sont jamais monodomaines (courbes situées au-dessus de celles des figures 2 et 3).

Un autre résultat théorique est confirmé par l'expérience : la dissymétrie due à l'anisotropie de forme. Sur l'exemple du grain i $\left(-49^{\circ}\right)$, on note que pour $\theta=-90^{\circ} \mathrm{E} \varphi_{1}=$ 0,47 alors que $E_{\varphi_{2}}=0,27$; expérimentalement on constate bien en effet que la germination dans un grain en aimantation directe se produit toujours pour une valeur de $\theta$ plus faible que lors de la germination dans un grain en aimantation inverse.

Il faut noter enfin que si les énergies de germination et de saturation se retrouvent aussi voisines c'est parce que les échantillons ont perdu leur coercitivité par suite de l'amincissement. Ce point fera l'objet d'un prochain article.

\section{Références}

[1] J. Bras, M. Fagot, J. Degauque, J. Microse. Spect. Elect. 13, 1988, 439-450

[2] F. C. Stoner, E.P. Wohlfarth, Phil. Trans., A 240, 1948, 599

[3] W. F. Jr.Brown, Single domain particles, Amer. J. Phys, 28, 1960, 542

[4] R. M. Bozorth, Ferromagnetism, (Van Nostrand), New York, 1951, 849

[5] J. Bras, J. Degauque, M. Fagot, K. Biyadi, J.M.M.M., 83, 1990, 203-205

[6] K. Biyadi, Thèse de l'Université Paul Sabatier, Toulouse, 1992 